\title{
Situación estimada de la zona cohesiva en el horno alto(*)
}

\author{
A. Cores*, J. Saiz de Ayala**, J. Mochon*, I. Ruiz-Bustinza* y R. Parra***
}

\begin{abstract}
Resumen En planta piloto se fabrica una serie de sinterizados a partir de mezclas minerales como las utilizadas en el horno alto. Se caracterizan los sinterizados mediante análisis químico y granulométrico y determinación de las temperaturas de reblandecimiento y fusión. En el horno alto, mediante una serie de sondas, se determinan las temperaturas en el horno, lo que permite situar la posición de la isoterma de mayor temperatura de $950^{\circ} \mathrm{C}$, que corresponde a la zona de reserva térmica. Se ha desarrollado un modelo que propone una estimación indirecta de la forma de la zona cohesiva a través de la determinación de la isoterma de mayor temperatura de la zona de reserva térmica. Los valores de las temperaturas de reblandecimiento y fusión de los sinterizados permiten estimar las isotermas que limitan la zona cohesiva, en el intervalo de $1.300-1.400^{\circ} \mathrm{C}$.
\end{abstract}

Palabras clave

Horno alto; Zona cohesiva; Zona de reserva térmica; Sinterización; Red de sistema neuronal.

\section{Forecast situation of the blast furnace cohesive zone}

Abstract

Keywords

\begin{abstract}
A series of sinters are manufactured in the pilot plant, using a mineral mixture, like that blast furnace. The sinters are characterised, by chemical and granulometric analysis and by determining the softening and melting temperatures. In the blast furnace temperatures are determined by using a series of probes in the furnace which make it possible to locate the position of the isotherm of higher temperature of $950^{\circ} \mathrm{C}$, which correspond to the reserve thermic zone. A model has been developed, proposing and indirect estimation of the shape of the cohesive zone through the determination of the isotherm with the highest temperature in the thermic reserve zone. The values of the softening and melting temperatures of sinters can be used to estimate the isotherms limiting the cohesive zone in the 1300 $1400^{\circ} \mathrm{C}$ range.
\end{abstract}

Blast furnace; Cohesive zone; Thermal reserve zone; Sintering; Neuronal network system.

\section{INTRODUCCIÓN}

En el tragante del horno alto se cargan y distribuyen capas alternadas de aglomerados de minerales de hierro (sínter y pelets) y coque y en su descenso por el horno el mineral de hierro se reduce, funde y gotea al crisol en donde se separan, por tener distinta densidad, el arrabio y la escoria formados.

La secuencia de la reducción de los óxidos de hierro a hierro metal es:

$$
\mathrm{Fe}_{2} \mathrm{O}_{3} \rightarrow \mathrm{Fe}_{3} \mathrm{O}_{4} \rightarrow \mathrm{FeO} \rightarrow \mathrm{Fe}
$$

Como agentes reductores actúan el carbono del coque y el $\mathrm{CO}$ que se produce en la combustión del coque:

- reducción directa: $\mathrm{Fe}_{\mathrm{x}} \mathrm{O}+\mathrm{C} \rightarrow \mathrm{x} \mathrm{Fe}+\mathrm{CO}$

- reducción indirecta: $\mathrm{Fe}_{\mathrm{x}} \mathrm{O}+\mathrm{CO} \rightarrow \mathrm{x} \mathrm{Fe}+$ $\mathrm{CO}_{2}$

El hierro que se produce en el horno alto se denomina arrabio y presenta en su composición, como elementos principales que acompañan al hierro, teniendo en cuenta los datos del análisis de los hornos europeos, 4,30-4,90 \% C y 0,35-0,90 \% Si.

A continuación del tragante se encuentra la cuba, zona del horno con temperaturas de 200 a $1.100^{\circ} \mathrm{C}$,

\footnotetext{
(•) Trabajo recibido el día 4 de marzo de 2009 y aceptado en su forma final el día 17 de noviembre de 2009.

* Centro Nacional de Investigaciones metalúrgicas, CEIM (CSIC), Avda. Gregorio del Amo, 8, 28040 Madrid.

** ARCELOR Corporación Siderúrgica. Apartado 570, 33280 Gijón

*** Universidad Concepción. Chile. alcores@cenim.csic.es; jesús.saizdeayala@arcelormittal.com; jmochon@cenim.csic.es; irbustinza@cenim.csic.es; rparra@udec.cl.
} 
en donde se inician las reacciones de reducción. Se extiende hasta el vientre, que es la zona del horno de mayor diámetro.

En la zona de reserva térmica del horno alto ${ }^{[1]}$ progresa, muy lentamente, la reducción del mineral iniciada en la zona superior del horno, por no ser favorables las condiciones existentes en esta parte del horno. La temperatura, en esta zona, es del orden de 900-950 ${ }^{\circ} \mathrm{C}$ y el hierro se encuentra, casi totalmente, en forma de FeO. Esta zona se localiza en la parte inferior de la cuba.

A continuación de la cuba, se encuentran los etalajes, que se extienden hasta la zona de toberas. En esta zona se completa toda la serie de reacciones del proceso. A través de esta zona el arrabio y la escoria gotean hacia el crisol. La zona inferior del horno es el crisol, donde periódicamente se cuelan el arrabio y la escoria por la piquera correspondiente.

La zona cohesiva ${ }^{[1}$ y 2$]$, descubierta cuando se llevó a cabo la disección de unos hornos altos ${ }^{[3}$ y 4$]$, está formada por capas alternas de mineral y coque que se encuentran en el intervalo de temperaturas comprendido entre $1.150^{\circ} \mathrm{C}$, en que empieza el reblandecimiento del mineral, luego, a mayor temperatura, en que pasa a un estado semifundido y, finalmente, hasta $1.400-1.500^{\circ} \mathrm{C}$, en que se funde ${ }^{[5]}$. El aire caliente (viento) soplado en las toberas, en su ascenso hacia el tragante del horno atraviesa la zona cohesiva. Lo hace con facilidad, por la capa de coque que es permeable y, con dificultad, por la capa mineral, debido a su estado pastoso ${ }^{[6]}$.

La reducción del mineral de hierro es parcial cuando alcanza la temperatura del reblandecimiento y aumenta a la temperatura de fusión y se completa cuando el mineral fundido atraviesa la zona de coque activo durante su descenso desde la zona cohesiva hacia el crisol.

La zona cohesiva desempeña un papel significativo en la distribución del flujo de gas ${ }^{[7]}$ y en el descenso de la carga en el horno ${ }^{[8]} \mathrm{y}$, con ello, en la operación eficiente de un horno alto. El viento, como se ha indicado, al llegar a la zona cohesiva, en su ascenso a través del horno, es desviado por las capas de reblandecimiento - fusión, impermeables al paso del gas, hacia las capas adyacentes de coque, que son permeables y distribuyen el gas hacia la zona granular, cerca de la pared del horno.

Los factores principales que controlan la localización y forma de la zona de reserva térmica y de las capas que componen la zona cohesiva son: la relación mineral/coque, la distribución de la carga en el tragante, la granulometría de los materiales que se cargan por el tragante y la profundidad y forma del raceway.
La localización más común se extiende desde los etalajes hasta la parte inferior de la cuba. La forma más común es la de una "V" invertida.

El presente trabajo se ha realizado con la colaboración ARCELOR - CENIM. La participación del CENIM ha consistido en la fabricación, en planta piloto, de una serie de sinterizados a partir de mezclas minerales que se utilizan en los dos hornos altos que la empresa ARCELOR tiene instalados en Gijón. Se determinan las temperaturas de reblandecimiento y fusión de los sinterizados y estas temperaturas darán una estimación de la localización de la zona cohesiva en el horno alto.

La participación de ARCELOR ha consistido en la instalación en el horno alto de una serie de sondas para la medida de la temperatura. Una red de sistema neuronal ayudará a caracterizar la forma de la zona de reserva térmica y de la zona cohesiva, haciendo uso de parámetros del proceso del horno como son perfiles de temperatura, medidas de presión y características puntuales de operación del horno.

\section{FABRICACIÓN DE SINTERIZADOS EN PLANTA PILOTO}

La empresa siderurgica ARCELOR tiene instaladas en Gijón dos plantas de sinterización de $200 \mathrm{~m}^{2}$ y $281 \mathrm{~m}^{2}$ de superficie efectiva de sinterización, que producen anualmente 5,0-5,5 millones de toneladas de sínter. Este sínter se utiliza como material de carga en los dos hornos altos (HA-A y HA-B), instalados en Gijón, de 11,3 m de diámetro de crisol y $2.349 \mathrm{~m}^{3}$ de volumen de trabajo, cada horno, que producen anualmente 3,8-4,3 millones de toneladas de arrabio.

En el presente trabajo, se han fabricado una serie de sinterizados en una planta piloto que el CENIM tiene instalada en ARCELOR, en Gijón. La planta piloto tiene una pila de 40 x $40 \mathrm{~cm}$ de sección transversal y $60 \mathrm{~cm}$ de altura y está equipada con un circuito de sección capaz de crear depresiones superiores a $1.470 \mathrm{MPa}$, un sistema de ignición de propano y un sistema para la medida del volumen de gas de entrada y la temperatura del gas de salida.

\subsection{Composición de las mezclas minerales}

En el CENIM se han utilizado una serie de minerales de hierro, fundentes (caliza y dunita) y coque, que habitualmente se consumen en las plantas de sinterización de 
SITUACIÓN ESTIMADA DE LA ZONA COHESIVA EN EL HORNO ALTO FORECAST SITUATION OF THE BLAST FURNACE COHESIVE ZONE

ARCELOR en Gijón. Se ha realizado un estudio muy amplio de estos minerales ${ }^{[9]}$.

La tabla I ofrece la composición de las mezclas minerales usadas para fabricar los sinterizados. La elección de estos minerales se ha hecho en base a motivos estratégicos, económicos y de calidad.

\subsection{Fabricación de sinterizados}

Se han fabricado una serie de sinterizados, en planta piloto, usando las mezclas minerales (Tabla I). En el proceso de fabricación se han mantenido constantes varios de los parámetros de operación, de acuerdo a la práctica industrial, tales como:

- Depresión de trabajo: $1470 \mathrm{MPa}$

- Aporte calorífico durante la ignición: $12.480 \mathrm{KJ}$

- Tiempo de ignición: 1,5 min

- Altura de sobreparrilla: $3 \mathrm{~cm}$

Mientras que otros parámetros han sido variables:

- Altura del lecho: 50 y $60 \mathrm{~cm}$

- Finos de retorno: 25 y $35 \%$

- Índice de basicidad $(\mathrm{CaO} / \mathrm{SiO} 2): 1,6$ y 1,9

En la tabla II se ofrecen las condiciones de fabricación de los sinterizados. El peso total de la mezcla

Tabla I. Composición de las mezclas minerales, \% en masa

Table I. Composition of ore mixtures, $\%$ mass

\begin{tabular}{lrccc}
\hline \multicolumn{1}{c}{ Mezclas } & $\mathbf{1}$ & $\mathbf{2}$ & $\mathbf{3}$ & $\mathbf{4}$ \\
\hline Andaluza & 4,0 & 10,3 & 10,3 & 10,3 \\
CVRD-Carajas & 25,0 & 27,8 & 21,3 & 24,4 \\
CVRD-Tubarao & - & - & 7,2 & 6,4 \\
San Isidro & 20,0 & 7,2 & - & - \\
Mount Wright & 8,0 & - & 7,2 & 7,2 \\
SNIM-Tazadit & 8,5 & 10,3 & - & - \\
SNIM-Normal & 4,0 & 6,2 & - & - \\
Goa Silicioso & 6,5 & - & 10,3 & 10,3 \\
Nimco & 4,5 & -- & - & - \\
Hamersley & 4,0 & 12,4 & - & 10,3 \\
MBR Brasil & - & 10,3 & 20,6 & 10,3 \\
Caliza & - & - & 7,9 & 6,1 \\
Lodos LD & 2,6 & 2,6 & 2,1 & 2,1 \\
Escoria LD & 3,0 & 3,0 & 2,1 & 2,1 \\
Chatarra de hierro & 1,3 & 1,3 & 2,1 & 2,1 \\
Polvo del tragante & 0,7 & 0,7 & 0,6 & 0,6 \\
Finos de sínter de & & & & \\
retorno & 7,9 & 7,9 & 8,1 & 8,1 \\
\hline
\end{tabular}

cargada en la paila aumenta con la altura del lecho, de 170 a $220 \mathrm{Kg}$.

Se ha realizado una caracterización muy amplia de estos sinterizados ${ }^{[10}$ y 11$]$.

\subsection{Temperaturas de reblandecimiento y fusión}

El ensayo para determinar las temperaturas de reblandecimiento (ST) y fusión (FT) se ha realizado utilizando el equipo LECO AF-600, con un horno que alcanza una temperatura máxima de $1.650^{\circ} \mathrm{C}$, en el cual se han fijado los parámetros siguientes con la ayuda de un ordenador: estándar ASTM con atmósfera inerte de nitrógeno, programa de inicio y fin de temperaturas, flujo de gas y velocidad de calentamiento[12].

\section{DETERMINACIÓN DE ISOTERMAS EN EL HORNO ALTO}

En la tabla III se ofrecen los datos de operación del horno alto $B$ durante el periodo en el que se ha realizado el presente trabajo ${ }^{[13]}$. Se puede apreciar una operación muy estable del horno.

En dicha tabla se ofrece la producción anual de arrabio del horno, que oscila entre 1,9 y 2,3 millones de toneladas. También se presenta el balance de los materiales alimentados y producidos por tonelada de arrabio (ta).

Los materiales de carga que alimentan el horno son los minerales de hierro, aglomerados de minerales de hierro (sínter y pelets) y coque, que se cargan por el tragante, a temperatura ambiente, en tamaños calibrados: mineral (15-30 mm), sínter $(12-35 \mathrm{~mm})$, pelets $(9-19 \mathrm{~mm})$ y coque $(25-45 \mathrm{~mm})$. El carbón pulverizado $(<0,1 \mathrm{~mm})$ se inyecta junto con el aire caliente (viento) por las toberas del horno. El horno produce arrabio y escoria, que se cuelan periódicamente por la piquera correspondiente, y gas que sale del horno por el tragante.

\subsection{Red de sistema neuronal e interfase gráfica de usuario}

En una primera etapa de la investigación se ha construido una red de sistema neuronal que ayudará a caracterizar en el horno la forma de la zona de reserva térmica y de la zona cohesiva, mediante el uso de los parámetros siguientes: presión, volumen, temperatura, humedad y enriquecimiento con oxígeno del 
Tabla II. Condiciones de operación para fabricar los sinterizados

Table II. Operation conditions to make sinters

\begin{tabular}{|c|c|c|c|c|c|c|c|c|c|c|}
\hline \multirow[b]{2}{*}{$\begin{array}{l}\text { Mezcla } \\
\text { mineral }\end{array}$} & \multirow[b]{2}{*}{ Sínter } & \multicolumn{6}{|c|}{ Material cargado en la paila, kg } & \multirow[b]{2}{*}{$\begin{array}{l}\mathrm{AL}, \\
\mathrm{cm}\end{array}$} & \multirow[b]{2}{*}{$\begin{array}{c}\text { FR, } \\
\%\end{array}$} & \multirow[b]{2}{*}{ B } \\
\hline & & $\begin{array}{c}\text { Peso de } \\
\text { mezcla } \\
\text { mineral }\end{array}$ & Dunita & Coque & $\begin{array}{c}\text { Finos } \\
\text { de } \\
\text { retorno }\end{array}$ & Caliza & Agua & & & \\
\hline \multirow{8}{*}{1} & $\mathrm{~S} 2$ & 124,0 & 2,0 & 5,4 & 30,0 & 9,6 & 9,5 & 50 & 25 & 1,6 \\
\hline & S4 & 113,6 & 1,9 & 4,7 & 27,5 & 13,2 & 8,8 & 50 & 25 & 1,9 \\
\hline & S6 & 113,5 & 1,7 & 4,2 & 38,5 & 9,9 & 8,8 & 50 & 35 & 1,6 \\
\hline & S8 & 113,5 & 1,6 & 4,4 & 38,5 & 13,0 & 8,8 & 50 & 35 & 1,9 \\
\hline & S10 & 139,4 & 2,6 & 5,9 & 38,8 & 12,3 & 10,0 & 60 & 25 & 1,6 \\
\hline & S12 & 134,3 & 2,6 & 5,7 & 32,5 & 16,1 & 9,4 & 60 & 25 & 1,9 \\
\hline & S14 & 134,3 & 2,3 & 5,3 & 45,5 & 11,9 & 10,0 & 60 & 35 & 1,6 \\
\hline & S16 & 129,1 & 2,0 & 5,1 & 43,8 & 16,4 & 10,5 & 60 & 35 & 1,9 \\
\hline \multirow{8}{*}{2} & S18 & 112,3 & 2,2 & 5,0 & 27,5 & 10,1 & 8,5 & 50 & 25 & 1,6 \\
\hline & S20 & 112,3 & 2,0 & 4,8 & 27,5 & 13,1 & 8,7 & 50 & 25 & 1,9 \\
\hline & S22 & 112,3 & 2,0 & 4,4 & 38,5 & 8,9 & 9,0 & 50 & 35 & 1,6 \\
\hline & S24 & 112,3 & 1,9 & 4,5 & 38,5 & 12,9 & 9,5 & 50 & 35 & 1,9 \\
\hline & S26 & 138,1 & 2,8 & 5,8 & 33,8 & 11,9 & 11,0 & 60 & 25 & 1,6 \\
\hline & S28 & 138,1 & 2,7 & 5,9 & 33,8 & 16,0 & 11,0 & 60 & 25 & 1,9 \\
\hline & S30 & 138,1 & 2,3 & 5,5 & 47,3 & 10,8 & 11,5 & 60 & 35 & 1,6 \\
\hline & S32 & 138,1 & 2,3 & 5,5 & 47,3 & 15,6 & 11,9 & 60 & 35 & 1,9 \\
\hline \multirow{5}{*}{3} & S33 & 135,0 & 3,7 & 5,9 & 33,8 & 16,8 & 12,0 & 60 & 25 & 1,9 \\
\hline & S34 & 140,0 & 3,5 & 6,0 & 35,0 & 16,7 & 12,0 & 60 & 25 & 1,9 \\
\hline & S35 & 136,0 & 3,4 & 5,4 & 34,0 & 10,1 & 12,0 & 60 & 25 & 1,9 \\
\hline & S36 & 136,0 & 3,3 & 5,1 & 34,0 & 10,3 & 12,0 & 60 & 25 & 1,9 \\
\hline & S37 & 136,0 & 3,7 & 5,1 & 34,0 & 10,2 & 12,0 & 60 & 25 & 1,9 \\
\hline \multirow{5}{*}{4} & S38 & 136,0 & 3,7 & 5,1 & 34,0 & 10,5 & 12,0 & 60 & 25 & 1,9 \\
\hline & S39 & 136,0 & 3,7 & 5,6 & 34,0 & 10,5 & 14,0 & 60 & 25 & 1,9 \\
\hline & S40 & 136,0 & 3,5 & 5,4 & 34,0 & 10,3 & 13,0 & 60 & 25 & 1,9 \\
\hline & S41 & 136,0 & 3,7 & 5,4 & 34,0 & 10,3 & 13,0 & 60 & 25 & 1,9 \\
\hline & S42 & 136,0 & 3,8 & 5,4 & 34,0 & 10,3 & 13,0 & 60 & 25 & 1,9 \\
\hline
\end{tabular}

$\mathrm{AL}=$ altura del lecho. $\mathrm{FR}=$ Finos de retorno. $\mathrm{B}=$ Basicidad $\left(\mathrm{CaO} / \mathrm{SiO}_{2}\right)$.

viento; consumo de coque; tasa de inyección de carbón pulverizado por toberas; contenido de silicio en el arrabio; contenidos de $\mathrm{CO}_{\text {y }} \mathrm{CO}_{2}$ y temperatura del gas de tragante y velocidad de carga.

La idea es clasificar las condiciones de operación de distintos modelos basados en la información disponible del proceso y vincular estos modelos con los que corresponden a diferentes perfiles de temperatura medidos con las sondas introducidas en la carga.

En la segunda etapa, se ha desarrollado un modelo que propone una estimación indirecta de la forma de la zona cohesiva, a través de la determinación de la isoterma de mayor temperatura (el límite superior) de la zona de reserva térmica. Este método hace uso de la información de la temperatura de las sondas situadas sobre la carga e introducidas en la carga, para el cálculo del gradiente de temperatura en la parte superior de la cuba, que permite obtener el límite superior de la zona de reserva térmica. Esta estimación indirecta de la zona cohesiva se puede justificar por los resultados de la disección de algunos hornos altos enfriados bruscamente que muestran que la forma de la zona cohesiva es similar a la forma de las isotermas que corresponden a la zona de reserva térmica (ejemplos los hornos altos Kukioka BF-4 y Hirohata BF-1 (Fig.1) ${ }^{[3 \text { y } 4]}$. 
Tabla III. Datos de operación del horno alto B

Table III. Operation data of blast furnace $B$

\begin{tabular}{|c|c|c|c|c|c|}
\hline \multicolumn{2}{|c|}{ Datos de operación } & \multirow{2}{*}{$\frac{2000}{2.127}$} & \multirow{2}{*}{$\begin{array}{c}2001 \\
2.095\end{array}$} & \multirow{2}{*}{$\frac{2002}{1.964}$} & \multirow{2}{*}{$\frac{2003}{2.264}$} \\
\hline Producción & $1000 t$ & & & & \\
\hline \multicolumn{6}{|l|}{ Carga } \\
\hline Sínter & $\mathrm{kg} / \mathrm{ta}$ & 1.101 & 1.110 & 1.154 & 1.215 \\
\hline Pelets & $\mathrm{kg} / \mathrm{ta}$ & 321 & 315 & 287 & 258 \\
\hline Minerales & $\mathrm{kg} / \mathrm{ta}$ & 169 & 163 & 149 & 129 \\
\hline \multicolumn{6}{|l|}{ Reductores sólidos } \\
\hline Coque & $\mathrm{kg} / \mathrm{ta}$ & 345 & 351 & 350 & 340 \\
\hline Carbón & $\mathrm{kg} / \mathrm{ta}$ & 141 & 131 & 132 & 159 \\
\hline \multicolumn{6}{|l|}{ Viento } \\
\hline Volumen & $1000 \mathrm{~m}^{3} \mathrm{STP} / \mathrm{h}$ & 248 & 244 & 246 & 249 \\
\hline Presión absoluta & bar & 4,30 & 4,30 & 4,40 & 4,30 \\
\hline Consumo específico de viento & $\mathrm{m}^{3} \mathrm{STP} / \mathrm{ta}$ & 946 & 958 & 942 & 929 \\
\hline Humedad total & $\mathrm{g} / \mathrm{m}^{3} \mathrm{STP}$ & 18,1 & 16,3 & 15,3 & 12,7 \\
\hline Oxígeno & $\%$ & 24,30 & 24,10 & 24,14 & 24,65 \\
\hline \multicolumn{6}{|l|}{ Volumen específico de oxígeno } \\
\hline para enriquecer el viento & $\mathrm{m}^{3} \mathrm{STP} / \mathrm{ta}$ & 41,0 & 39,3 & 39,0 & 45,0 \\
\hline Temperatura del viento & ${ }^{\circ} \mathrm{C}$ & 1.193 & 1.181 & 1.192 & 1.211 \\
\hline Temperatura adiabática de llama & ${ }^{\circ} \mathrm{C}$ & 2.218 & 2.243 & 2.250 & 2.223 \\
\hline \multicolumn{6}{|l|}{ Arrabio } \\
\hline C & $\%$ & 4,74 & 4,75 & 4,74 & 4,70 \\
\hline $\mathrm{Si}$ & $\%$ & 0,45 & 0,46 & 0,44 & 0,36 \\
\hline $\mathrm{Mn}$ & $\%$ & 0,47 & 0,40 & 0,41 & 0,47 \\
\hline$P$ & $\%$ & 0,066 & 0,070 & 0,072 & 0,077 \\
\hline$S$ & $\%$ & 0,030 & 0,030 & 0,026 & 0,026 \\
\hline Temperatura & ${ }^{\circ} \mathrm{C}$ & 1.481 & 1.481 & 1.479 & 1.478 \\
\hline \multicolumn{6}{|l|}{ Escoria } \\
\hline Volumen & $\mathrm{kg} / \mathrm{ta}$ & 257 & 250 & 262 & 270 \\
\hline $\mathrm{CaO}$ & $\%$ & 40,70 & 40,50 & 41,13 & 40,15 \\
\hline $\mathrm{MgO}$ & $\%$ & 8,40 & 8,30 & 8,36 & 8,65 \\
\hline $\mathrm{Al}_{2} \mathrm{O}_{3}$ & $\%$ & 11,80 & 12,50 & 12,01 & 13,18 \\
\hline $\mathrm{SiO}_{2}$ & $\%$ & 34,70 & 34,90 & 34,79 & 34,38 \\
\hline$S^{2}$ & $\%$ & 0,80 & 0,80 & 0,78 & 0,68 \\
\hline $\mathrm{K}_{2} \mathrm{O}+\mathrm{Na}_{2} \mathrm{O}$ & $\%$ & 0,83 & 0,79 & 0,80 & 0,97 \\
\hline \multicolumn{6}{|l|}{ Gas del tragante } \\
\hline $\mathrm{CO}$ & $\%$ & 23,0 & 23,3 & 22,9 & 23,6 \\
\hline $\mathrm{CO}_{2}$ & $\%$ & 22,0 & 22,1 & 21,5 & 22,4 \\
\hline $\mathrm{H}_{2}$ & $\%$ & 3,6 & 3,3 & 3,8 & 3,5 \\
\hline Presión absoluta & bar & 2,89 & 2,80 & 2,91 & 2,91 \\
\hline Volumen & $\mathrm{m}^{3} \mathrm{STP} / \mathrm{ta}$ & 1.393 & 1.417 & 1.396 & 1.385 \\
\hline Temperatura & ${ }^{\circ} \mathrm{C}$ & 143 & 145 & 145 & 129 \\
\hline
\end{tabular}

ta $=$ tonelada de arrabio. 


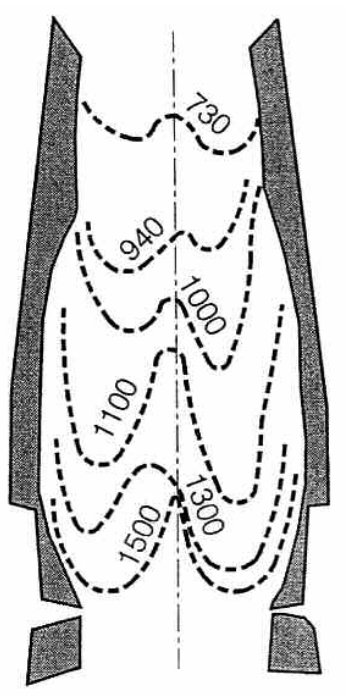

KUKIOKA N 4

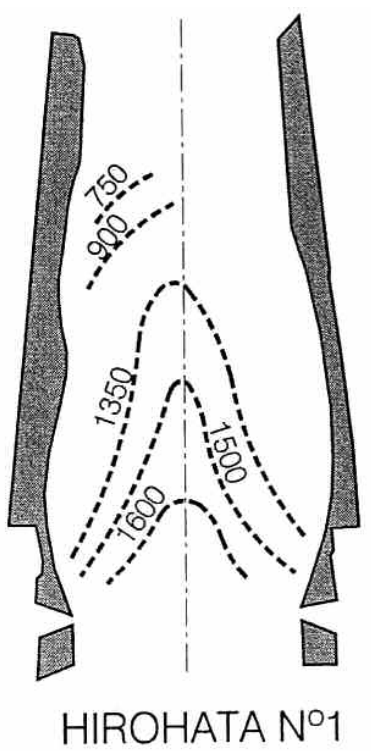

Figura 1. Similitud de la forma de las isotermas que delimitan el límite superior de la zona de reserva térmica $\left(\sim 950^{\circ} \mathrm{C}\right)$ y de la zona cohesiva $\left(\sim 1300-1500^{\circ} \mathrm{C}\right)$.

Figure 1. Similarity of the shape of the isotherms delimiting the top of the thermal reserve zone $\left(\sim 950^{\circ} \mathrm{C}\right)$ and the cohesive zone ( 1300$\left.1500^{\circ} \mathrm{C}\right)$.

El horno Kukioka BF-4, diseccionado en 1970, tiene una altura de $31 \mathrm{~m}$, un volumen interno de $1.407 \mathrm{~m}^{3}$ y un diámetro de crisol de $7,9 \mathrm{~m}$. El horno Hirohata BF-1, diseccionado en 1971, tiene una altura de $28 \mathrm{~m}$, un volumen interno de $1.279 \mathrm{~m}^{3} \mathrm{y}$ un diámetro de crisol de 8,5 m. El horno alto B de Gijón presenta las siguientes dimensiones: altura $36,7 \mathrm{~m}$, volumen interno, $2.349 \mathrm{~m}^{3}$ y diámetro de crisol, 11,3 m. El horno de Gijón es mayor que los hornos japoneses, aunque las alturas se pueden considerar del mismo orden, lo que permite suponer que se puede extrapolar la operación de los hornos japoneses al horno de Gijón.

Se ha desarrollado una interfase gráfica de usuario que muestra la estimación de la posición del límite superior de la zona de reserva térmica y de otras variables destacadas.

\subsection{Evaluación de los datos de las variables medidas en el horno alto $B$}

Durante el período de mayo 2000 a mayo 2002, se han utilizado 15 conjuntos de datos con una velocidad de toma de muestra de $2 \mathrm{~min}$, cada uno, cubriendo el período de un mes, lo que supone 2.200 muestras. Las variables medidas se han clasificado en dos grupos:

- Variables de operación.

- Temperaturas sobre la carga y en la carga.

\subsubsection{Variables de operación}

Este grupo se ha establecido con las siguientes variables: humedad, presión, temperatura y volumen de viento; tasa de inyección de carbón pulverizado; consumo de coque; contenido de silicio en el arrabio; contenidos de $\mathrm{CO}_{\text {y }} \mathrm{CO}_{2}$ en el gas del tragante y velocidad de carga.

Se ha aplicado la autoorganización de los mapas característicos de las redes neuronales para clasificar este grupo de datos en 10 modelos. Sus propiedades son las siguientes:

- Número de neurones (modelos): 10.

- Número de epochs: 10. Es el número de veces que toda la colección de la muestra entra en el proceso de entrenamiento.

- Coeficiente inicial de aprendizaje: 0,05. Este parámetro disminuirá para cada nuevo epoch, dividiendo el coeficiente inicial entre el número de epoch respectivo.

- Coeficiente de vecindad: 0,1.

- Normalización de los datos de la matriz. Cada elemento se divide entre el máximo de la variable respectiva. Como resultado la matriz presenta valores de 0 a 10 y cada variable posee el mismo peso. Cuando termina el entrenamiento se obtiene una matriz modelo y se realiza una acción inversa de normalización, para que las variables posean los órdenes de magnitudes iniciales. Cuando se obtiene una matriz modelo se realiza la validación y cada perfil del grupo de datos estará asociado con el modelo.

La tabla IV muestra los modelos obtenidos para el grupo de datos de las variables de operación.

El primer modelo está relacionado con un estado de interrupción de la marcha del horno y los dos últimos con un funcionamiento ideal. El segundo y el tercero se asocian con las etapas de arranque o parada del horno, o cambios realizados en el enriquecimiento del viento con oxígeno y en la inyección de carbón pulverizado por toberas.

\subsubsection{Medidas de temperaturas sobre la carga y en la carga}

La señal de medida por la sonda, sobre la carga, ha estado muy alterada debido al sistema de refrigeración 
SITUACIÓN ESTIMADA DE LA ZONA COHESIVA EN EL HORNO ALTO FORECAST SITUATION OF THE BLAST FURNACE COHESIVE ZONE

Tabla IV. Distribución de las variables de operación

Table IV. Operation variables patterns

\begin{tabular}{|c|c|c|c|c|c|c|c|c|c|c|}
\hline & \multicolumn{10}{|c|}{ Distribución } \\
\hline & 1 & 2 & 3 & 4 & 5 & 6 & 7 & 8 & 9 & 10 \\
\hline \multicolumn{11}{|l|}{ Presión del viento } \\
\hline $\begin{array}{l}\text { Velocidad de carga } \\
\text { (cargas/h) } \\
\text { Temperatura media del }\end{array}$ & 0,02 & 2,35 & 4,24 & 5,77 & 5,48 & 5,54 & 5,82 & 5,49 & 5,60 & 11,41 \\
\hline $\begin{array}{l}\text { gas del tragante }\left({ }^{\circ} \mathrm{C}\right) \\
\text { Contenido de } \mathrm{CO}_{2} \text { en el }\end{array}$ & 3 & 158 & 20,5 & 133 & 131 & 149 & 115 & 123 & 140 & 322 \\
\hline $\begin{array}{l}\text { gas del tragante }(\%) \\
\text { Contenido de } \mathrm{CO} \text { en el }\end{array}$ & 0,13 & 11,24 & 18,52 & 19,57 & 21,46 & 23,28 & 22,25 & 23,09 & 22,55 & 26,46 \\
\hline gas del tragante $(\%)$ & 0,12 & 11,93 & 23,55 & 22,86 & 22,57 & 22,46 & 22,56 & 23,09 & 23,12 & 31,96 \\
\hline $\begin{array}{l}\text { Contenido de Si en el } \\
\text { arrabio (\%) }\end{array}$ & 0,00 & 0,45 & 0,89 & 0,37 & 0,48 & 0,47 & 0,39 & 0,37 & 0,53 & 1,66 \\
\hline $\begin{array}{l}\text { Consumo de coque } \\
(\mathrm{kg} / \mathrm{ta})\end{array}$ & 4 & 461 & 499 & 473 & 381 & 330 & 354 & 330 & 329 & 1.215 \\
\hline $\begin{array}{l}\text { Volumen del viento } \\
\left(\mathrm{m}^{3} / \mathrm{h}\right) \cdot 10^{3} \\
\text { Temperatura del viento }\end{array}$ & 0,567 & 91,1 & 236,0 & 256,5 & 258,3 & 260,3 & 261,3 & 261,8 & 261,9 & 275,4 \\
\hline $\begin{array}{l}\left({ }^{\circ} \mathrm{C}\right) \\
\text { Enriquecimiento en }\end{array}$ & 10 & 660 & 1.019 & 1.067 & 1.160 & 35 & 1.211 & 1.220 & 1.222 & 1.235 \\
\hline $\begin{array}{l}\mathrm{O}_{2}\left(\mathrm{~m}^{3} / \mathrm{h}\right) \\
\text { Inyección de carbón }\end{array}$ & 0 & 73 & 506 & 278 & 4.782 & 12.450 & 10.382 & 13.706 & 13.634 & 14.817 \\
\hline $\begin{array}{l}\text { pulverizado (t/h) } \\
\text { Humedad del viento }\end{array}$ & 0,00 & 0,37 & 3,39 & 2,52 & 22,30 & 43,95 & 38,17 & 46,07 & 43,31 & 52,42 \\
\hline$\left(\mathrm{g} / \mathrm{m}^{3}\right)$ & 0,08 & 10,71 & 26,86 & 11,42 & 15,49 & 20,30 & 12,80 & 11,24 & 27,63 & 56,43 \\
\hline
\end{tabular}

ta $=$ tonelada de arrabio.

por nitrógeno, para evitar el deterioro de la sonda, durante la adquisición de la primera serie de datos (mayo 2000 - mayo 2001). Las medidas principales alteradas son las medidas de los termopares centrales. Por esta razón, se han eliminado la mayoría de las medidas centrales de los 6 puntos de la sonda. Por tanto, se ha utilizado la señal tomada por el resto de los termopares y se han obtenido los nuevos datos de cada perfil central mediante la interpolación de las medidas restantes. Este problema se ha resuelto en la última serie de datos, debido a un nuevo sistema de refrigeración que no altera las medidas de las sondas centrales.

La sonda en la carga está localizada a una altura 29,45 m, es decir, 7,17 m debajo de la localización de la sonda sobre la carga. Debido a su manejo complejo, esta sonda se ha podido utilizar pocas veces para realizar medidas durante este trabajo: 56 medidas de mayo 2001 a mayo 2002.
En la figura 2 se muestra la localización de las sondas en el horno.

\section{RESULTADOS}

\subsection{Sinterizados}

En la tabla V se ofrece el análisis químico y las temperaturas de reblandecimiento y fusión de los sinterizados fabricados.

Se puede observar una composición bastante uniforme a pesar de la naturaleza distinta de los minerales utilizados y de las condiciones de operación.

Las temperaturas de fusión son elevadas y la diferencia para cada sinterizado, entre la temperatura de fusión y el reblandecimiento, es pequeña. A partir de estas temperaturas, se puede estimar en $1.300^{\circ} \mathrm{C}$ 


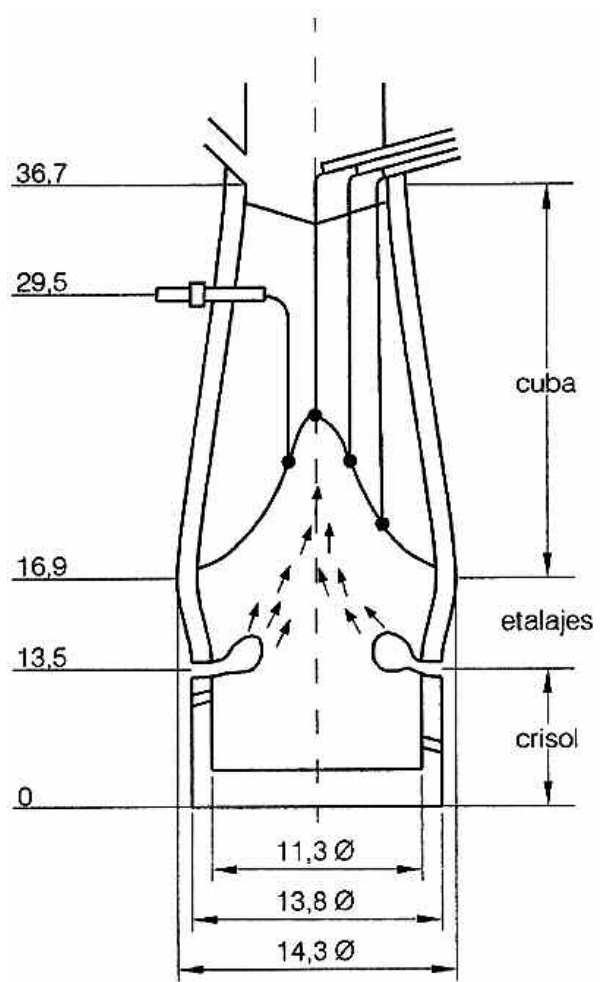

Figura 2. Localización de las diferentes sondas en el horno alto B. Cotas en metros.

Figure 2. Location of different probes at blast furnace $B$. Height in meters.

el valor inferior y en $1.400{ }^{\circ} \mathrm{C}$ el valor superior de las isotermas que limitan la zona cohesiva. Estas condiciones son beneficiosas para una buena situación de la zona cohesiva y una buena marcha del horno.

En la tabla VI se ofrece el análisis granulométrico de los sinterizados. Se observa que el mayor peso corresponde a la fracción mayor de $40 \mathrm{~mm}$. La fracción de (12 - 5) mm y la fracción menor de $5 \mathrm{~mm}$ presentan un peso similar, excepto para los sinterizados S14 y S30. La fracción menor de $5 \mathrm{~mm}$, que se denomina "finos de retorno", presenta un porcentaje considerable del peso total del sínter. Estos finos de retorno forman parte de la composición de las mezclas minerales que van a experimentar el proceso de su sinterización (Tablas I y II).

\subsection{Modelo para situar el límite superior de la zona de reserva térmica}

La figura 3 presenta los valores tomados por las sondas sobre la carga y en la carga a las 13:06 h del 17 de agosto de 2001.
La figura 4 muestra el aumento de temperatura por metro entre los termopares 1 y 6 de cada sonda, suponiendo que la temperatura no cambia en el nivel superior de la carga.

El gradiente de temperatura en el tragante, para cada termopar en la línea vertical, se calcula como indica la ecuación siguiente:

$$
\frac{\Delta \text { Temp. }}{\Delta \text { Distancia }}=\frac{\text { Temp. de la sonda sobre la carga }- \text { Temp. en la carga }}{\text { Altura de la carga }- \text { Altura de la sondaen la carga }}
$$

La figura 5 muestra la variación de temperaturas entre los termopares de las sondas sobre y en la carga, como función de la distancia entre el nivel de carga y la posición de la sonda en la carga. Se ha obtenido un valor medio del gradiente de la temperatura para cada termopar en la línea vertical.

$$
\begin{array}{lll}
=>\text { Termopar } & 1: 35,0^{\circ} \mathrm{C} / \mathrm{m} \\
=> & \text { id } & 2: 43,5^{\circ} \mathrm{C} / \mathrm{m} \\
=> & \text { id } & 3: 73,7^{\circ} \mathrm{C} / \mathrm{m} \\
=> & \text { id } & 4: 84,1^{\circ} \mathrm{C} / \mathrm{m} \\
=> & \text { id } & 5: 91,3^{\circ} \mathrm{C} / \mathrm{m} \\
=> & \text { id } & 6: 90,6^{\circ} \mathrm{C} / \mathrm{m}
\end{array}
$$

Al utilizar estos valores se ha desarrollado un modelo para la determinación de la posición del límite superior de la zona de reserva térmica, para cada medida sobre la carga y en la carga, y para cada nivel de profundidad de la carga, al aplicar la ecuación siguiente:

$$
L S Z R T=A H-P C-\frac{900-T S C}{\Delta T}
$$

LSZRT: Límite superior de la zona de reserva térmica $(\mathrm{m})$

AH: Altura del horno $=36,71 \mathrm{~m}$

PC: Profundidad de la carga $(\mathrm{m})$

TSC: Temperatura sobre la carga ${ }^{\circ} \mathrm{C}$

$\Delta \mathrm{T}$ : Gradiente de temperatura $\left({ }^{\circ} \mathrm{C} / \mathrm{m}\right)$

La figura 6 muestra la altura estimada del límite superior de la zona de reserva térmica en la línea vertical del termopar 5, para un período de 100 muestras.

\subsection{Interfase gráfica de usuario}

Se ha desarrollado una interfase gráfica de usuario que estima la posición del límite superior de la zona de reserva térmica y otras variables destacadas, usando una interfase gráfica de usuario de MATLAB. 
SITUACIÓN ESTIMADA DE LA ZONA COHESIVA EN EL HORNO ALTO FORECAST SITUATION OF THE BLAST FURNACE COHESIVE ZONE

Tabla V. Análisis químico (\% en masa) y temperaturas $\left({ }^{\circ} \mathrm{C}\right)$ de reblandecimiento $(\mathrm{ST})$ y fusión (FT) de los sinterizados

Table V. Chemical analysis (\% mass) and softening (ST) and melting (FT) temperatures ( $\left.{ }^{\circ} \mathrm{C}\right)$ of sinters

\begin{tabular}{|c|c|c|c|c|c|c|c|c|c|c|c|c|c|c|}
\hline $\begin{array}{l}\text { Mezcla } \\
\text { mineral }\end{array}$ & Sínter & $\begin{array}{l}\mathrm{Fe} \\
\text { total }\end{array}$ & $\mathrm{FeO}$ & $\mathrm{SiO}_{2}$ & $\mathrm{Al}_{2} \mathrm{O}_{3}$ & $\mathrm{CaO}$ & MgO & $\mathrm{MnO}$ & $S$ & $\mathbf{P}$ & $\mathrm{Na}_{2} \mathrm{O}$ & $\mathrm{K}_{2} \mathrm{O}$ & ST & FT \\
\hline \multirow{8}{*}{1} & S2 & 57,5 & 5,5 & 5,7 & 1,16 & 9,15 & 1,55 & 0,64 & 0,012 & 0,034 & 0,041 & 0,066 & 1.329 & 1.350 \\
\hline & S4 & 56,3 & 5,7 & 5,6 & 1,35 & 10,45 & 1,71 & 0,72 & 0,013 & 0,041 & 0,038 & 0,060 & 1.324 & 1.348 \\
\hline & S6 & 57,1 & 3,5 & 5,6 & 1,17 & 9,25 & 1,63 & 0,66 & 0,010 & 0,030 & 0,039 & 0,064 & 1.329 & 1.355 \\
\hline & S8 & 56,0 & 3,2 & 5,4 & 1,15 & 11,10 & 1,53 & 0,64 & 0,017 & 0,031 & 0,038 & 0,065 & 1.316 & 1.340 \\
\hline & S10 & 57,1 & 5,0 & 5,8 & $3 \quad 1,24$ & 9,20 & 1,44 & 0,65 & 0,012 & 0,041 & 0,038 & 0,058 & 1.352 & 1.366 \\
\hline & $\mathrm{S} 12$ & 56,2 & 4,6 & 5,7 & 1,15 & 10,90 & 1,58 & 0,68 & 0,015 & 0,039 & 0,039 & 0,058 & 1.348 & 1.362 \\
\hline & S14 & 57,5 & 3,3 & 5,4 & 1,20 & 9,10 & 1,51 & 0,66 & 0,011 & 0,038 & 0,037 & 0,060 & 1.354 & 1.370 \\
\hline & S16 & 56,7 & 4,3 & 5,5 & 1,17 & 10,40 & 1,50 & 0,70 & 0,018 & 0,040 & 0,037 & 0,056 & 1.343 & 1.359 \\
\hline \multirow{8}{*}{2} & S18 & 57,3 & 5,7 & 5,4 & 1,22 & 9,35 & 1,70 & 0,74 & 0,011 & 0,042 & 0,039 & 0,071 & 1.325 & 1.348 \\
\hline & S20 & 56,6 & 5,6 & 5,3 & 1,17 & 10,50 & 1,68 & 0,72 & 0,014 & 0,039 & 0,037 & 0,071 & 1.312 & 1.338 \\
\hline & S22 & 57,6 & 3,7 & 5,4 & 1,19 & 8,80 & 1,60 & 0,80 & 0,013 & 0,040 & 0,036 & 0,072 & 1.347 & 1.364 \\
\hline & S24 & 56,9 & 3,6 & 4,9 & 1,16 & 10,35 & 1,61 & 0,71 & 0,018 & 0,040 & 0,031 & 0,068 & 1.316 & 1.350 \\
\hline & S26 & 58,0 & 4,7 & 5,2 & ? 1,20 & 8,60 & 1,62 & 0,75 & 0,012 & 0,040 & 0,032 & 0,066 & 1.342 & 1.352 \\
\hline & S28 & 56,5 & 5,0 & 5,7 & 1,10 & 10,13 & 1,66 & 0,73 & 0,014 & 0,036 & 0,036 & 0,072 & 1.322 & 1.343 \\
\hline & S30 & 56,7 & 3,5 & 5,0 & 1,19 & 9,05 & 1,56 & 0,79 & 0,012 & 0,041 & 0,040 & 0,075 & 1.336 & 1.352 \\
\hline & S32 & 57,1 & 3,9 & 5,8 & 1,10 & 9,20 & 1,45 & 0,69 & 0,015 & 0,038 & 0,039 & 0,072 & 1.341 & 1.355 \\
\hline \multirow{5}{*}{3} & S33 & 5 & 0 & , & 1,02 & 9,15 & 1,75 & 0,96 & 0,013 & 0,036 & 0,036 & 0,065 & - & - \\
\hline & S34 & 55,5 & 5,2 & 5,5 & $5 \quad 0,99$ & 10,85 & 1,65 & 0,96 & 0,022 & 0,030 & 0,042 & 0,070 & - & - \\
\hline & S35 & 56,2 & 5,5 & 5,7 & 0,91 & 10,85 & 1,55 & 0,94 & 0,017 & 0,031 & 0,038 & 0,064 & 1.335 & 1.365 \\
\hline & S36 & 56,0 & 3,8 & 5,6 & 1,06 & 10,80 & 1,65 & 0,93 & 0,023 & 0,035 & 0,045 & 0,078 & 1.327 & 1.358 \\
\hline & S37 & 55,4 & 3,9 & 5,8 & $3 \quad 1,11$ & 11,10 & 1,68 & 0,99 & 0,020 & 0,029 & 0,042 & 0,078 & 1.321 & 1.351 \\
\hline \multirow{5}{*}{4} & S38 & 54,9 & 3,5 & 6,0 & 1,15 & 11,55 & 1,68 & 0,93 & 0,025 & 0,032 & 0,041 & 0,079 & 1.334 & 1.363 \\
\hline & S39 & 55,7 & 5,5 & 5,6 & 1,12 & 11,55 & 1,88 & 0,85 & 0,015 & 0,035 & 0,041 & 0,092 & - & - \\
\hline & S40 & 56,1 & 5,3 & 5,3 & $3 \quad 1,18$ & 10,10 & 1,70 & 0,80 & 0,012 & 0,034 & 0,039 & 0,091 & 1.329 & 1.357 \\
\hline & S41 & 56,5 & 5,2 & 5,2 & ? 1,22 & 10,10 & 1,69 & 0,97 & 0,014 & 0,032 & 0,042 & 0,088 & - & - \\
\hline & S42 & 55,3 & 4,4 & 5,7 & 1,27 & 11,10 & 2,01 & 0,80 & 0,015 & 0,037 & 0,044 & 0,095 & 1.327 & 1.349 \\
\hline
\end{tabular}

Se dispone de dos versiones de interfase que serán instrumentos en la sala de control del horno alto. La primera, una interfase en línea, permitirá al operador ver la posición estimada del límite superior de la zona de reserva térmica en tiempo real. La segunda, una interfase off-line, permitirá al operador cambiar la velocidad de observación. En ambos casos, el usuario puede ver la gráfica del límite superior de la zona de reserva térmica para cada muestra. Además, se pueden ver las gráficas de, al menos, 20 variables destacadas.

Las figuras 7 y 8 muestran las pantallas de la interfase gráfica de usuario off-line. La primera es una pantalla de un modo interrumpido y la segunda es una pantalla de un modo continuo. La figura 9 muestra la pantalla de la interfase en línea.

\section{DISCUSIÓN}

\subsection{Zona cohesiva}

El sínter es el principal aglomerado de minerales de hierro que se utiliza como material de carga en el horno alto. Su calidad es muy importante para la buena marcha del horno ${ }^{[14]}$.

El funcionamiento de un horno alto está condicionado por la geometría y situación de la zona cohesiva que está delimitada por las isotermas de reblan-

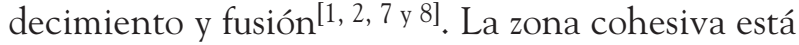
constituida por capas alternativas de sinterizado en estado pastoso y coque. Son estas últimas, denominadas "ventanas de coque", las que permiten el paso del 
Tabla VI. Análisis granulométrico de los sinterizados fabricados

Tabla VI. Granulometric analysis of sinters made

\begin{tabular}{|c|c|c|c|c|c|c|}
\hline \multirow{2}{*}{$\begin{array}{l}\text { Mezcla } \\
\text { mineral }\end{array}$} & \multirow{2}{*}{ Sínter } & \multicolumn{5}{|c|}{$\begin{array}{c}\text { Peso de sínter que corresponde a cada fracción } \\
\text { granulométrica, } \mathrm{kg}\end{array}$} \\
\hline & & $>40 \mathrm{~mm}$ & $(40-20) \mathrm{mm}$ & $(20-12) \mathrm{mm}$ & $(12-5) \mathrm{mm}$ & $<5 \mathrm{~mm}$ \\
\hline \multirow{8}{*}{1} & S2 & 48,9 & 17,2 & 8,8 & 31,3 & 28,6 \\
\hline & S4 & 35,8 & 29,6 & 12,4 & 28,1 & 26,1 \\
\hline & S6 & 36,9 & 21,6 & 8,3 & 33,3 & 36,2 \\
\hline & S8 & 35,5 & 23,4 & 9,3 & 31,0 & 34,4 \\
\hline & S10 & 61,2 & 26,2 & 12,0 & 32,5 & 32,6 \\
\hline & S12 & 54,5 & 34,5 & 12,3 & 28,0 & 31,0 \\
\hline & S14 & 56,7 & 23,0 & 8,2 & 33,6 & 42,0 \\
\hline & S16 & 47,8 & 27,3 & 11,6 & 36,6 & 40,0 \\
\hline \multirow{8}{*}{2} & S18 & 43,7 & 27,3 & 9,9 & 25,8 & 25,2 \\
\hline & S20 & 38,0 & 33,2 & 11,2 & 24,3 & 24,8 \\
\hline & S22 & 38,9 & 20,8 & 7,8 & 31,9 & 37,2 \\
\hline & S24 & 36,7 & 24,8 & 9,2 & 29,2 & 33,7 \\
\hline & S26 & 57,6 & 27,4 & 11,5 & 34,6 & 33,8 \\
\hline & S28 & 42,0 & 37,8 & 14,5 & 33,7 & 32,6 \\
\hline & S30 & 55,1 & 23,3 & 8,5 & 34,4 & 46,5 \\
\hline & S32 & 51,1 & 30,6 & 10,0 & 33,6 & 37,0 \\
\hline \multirow{4}{*}{3} & S34 & 52,4 & 39,1 & 13,1 & 27,4 & 25,5 \\
\hline & S35 & 42,9 & 39,7 & 12,9 & 30,3 & 28,5 \\
\hline & S36 & 52,0 & 34,4 & 12,5 & 31,8 & 32,0 \\
\hline & S37 & 50,2 & 36,2 & 13,5 & 32,2 & 31,7 \\
\hline \multirow{5}{*}{4} & S38 & 43,0 & 29,7 & 12,3 & 37,1 & 39,1 \\
\hline & S39 & 45,0 & 37,5 & 13,3 & 31,0 & 28,9 \\
\hline & S40 & 38,7 & 36,3 & 15,0 & 36,0 & 32,9 \\
\hline & S41 & 47,9 & 32,7 & 12,3 & 33,3 & 32,2 \\
\hline & S42 & 47,0 & 31,8 & 13,0 & 34,1 & 32,8 \\
\hline
\end{tabular}

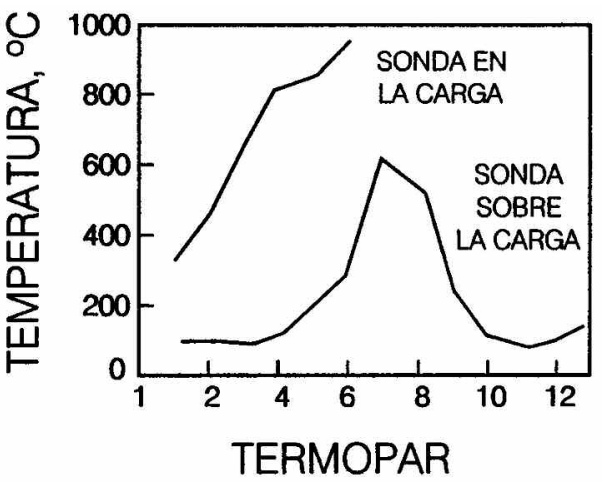

Figura 3. Temperatura en la carga y sobre la carga.

Figure 3. In-burden and above burden temperatures. gas reductor hacia las zonas superiores del horno. Es, por tanto, importante que la zona cohesiva sea lo más estrecha posible para facilitar el paso del gas y que esté situada lo más bajo posible en el horno para que la zona de preparación del horno, situada encima de la zona cohesiva, tenga extensión suficiente para permitir la reducción de los óxidos de hierro. Para conseguir ambos condicionantes, es necesario que las temperaturas ST y FT sean lo más altas posibles y que la diferencia FT-ST sea mínima. Esto, se ha conseguido con los sinterizados fabricados (Tabla V) que permiten situar las isotermas de la zona cohesiva en el intervalo de $1.300-1.400^{\circ} \mathrm{C}$.

En el horno alto Kokura BF-2, diseccionado en 1974, se han contabilizado 32 capas de reblandecimiento y fusión, alternando con capas de coque ${ }^{[15]}$. 

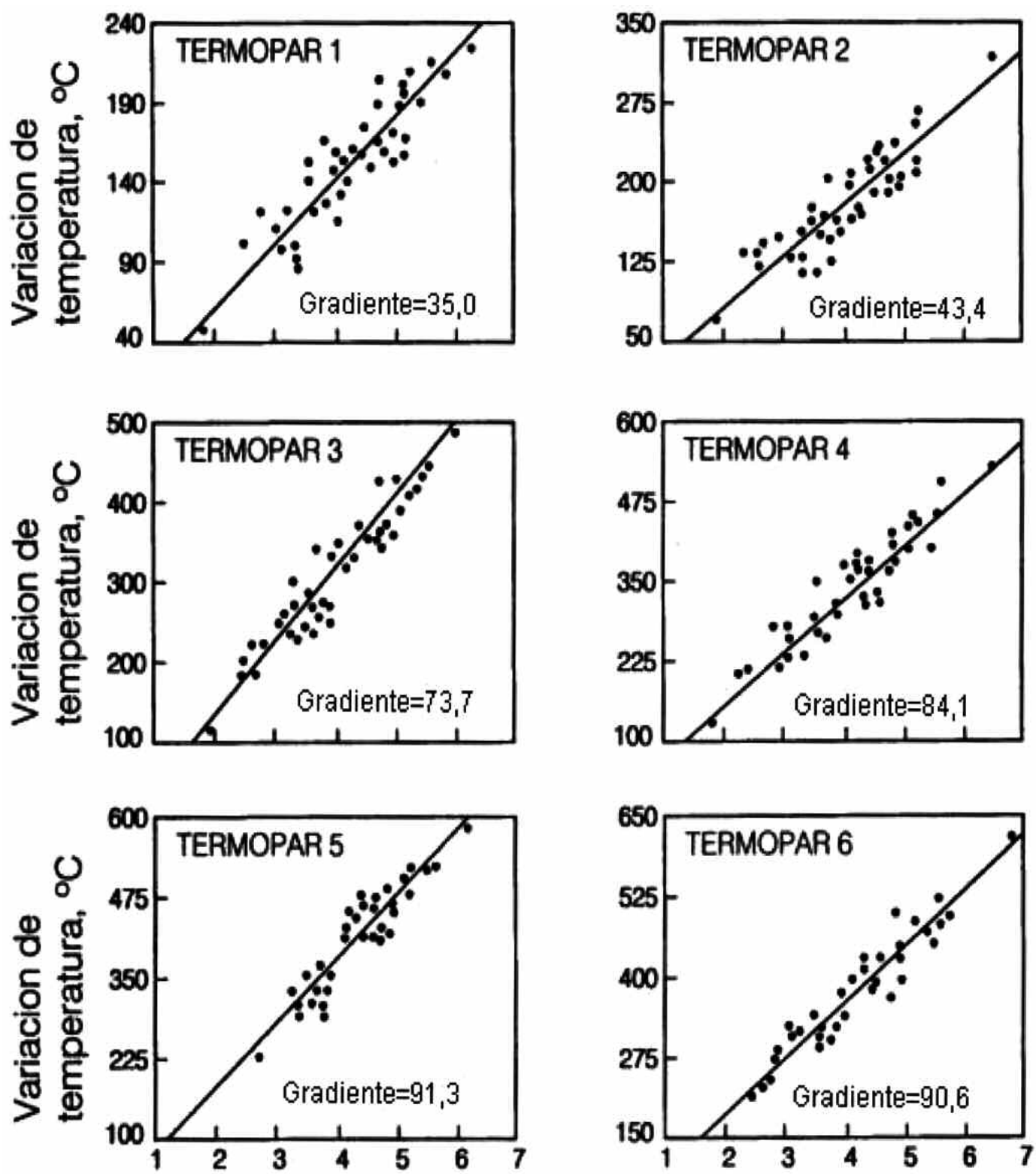

Figura 4. Variación de temperatura entre los termopares 1 y 6 de cada sonda.

Figure 4. Temperature variation between thermocouples 1 and 6 of each probe.

En la tabla VII se ofrecen las dimensiones de este horno. Las capas de reblandecimiento y fusión están formadas por gránulos de aglomerado de minerales de hierro que, al principio, experimentan una ligera sinterización y terminan con un goteo hacia el crisol. Estas capas de aglomerado presentan un grado de reducción elevado de los óxidos de hierro.

En la tabla $\mathrm{V}$ se observa que el contenido en alúmina es bajo en todos los sinterizados $(<1,40 \%)$, lo cual favorece su mejor calidad ${ }^{[16]}$. Los contenidos en $\mathrm{MgO}(<2,10 \%)$ y en $\mathrm{FeO}(<6 \%)$, también se pueden considerar bajos. Los contenidos en Fe total (55$58 \%), \mathrm{SiO}_{2}(5-6 \%)$ y $\mathrm{CaO}(8,5-11,5 \%)$ se pueden considerar normales. Se ha investigado que un contenido bajo en $\mathrm{SiO}_{2}$ y en $\mathrm{CaO}$ y alto en $\mathrm{MgO}$ y en $\mathrm{FeO}$, favorece un buen comportamiento del sinterizado frente a su reblandecimiento, y que el contenido en $\mathrm{Al}_{2} \mathrm{O}_{3}$ tiene poca influencia sobre el reblandecimiento ${ }^{[17]}$.

\subsection{Zona de reserva térmica}

Se ha desarrollado un modelo de horno alto que propone una estimación indirecta de la forma de la zona cohesiva a través de la determinación del límite superior de la zona de reserva térmica. Este método emplea la información de las temperaturas de las 


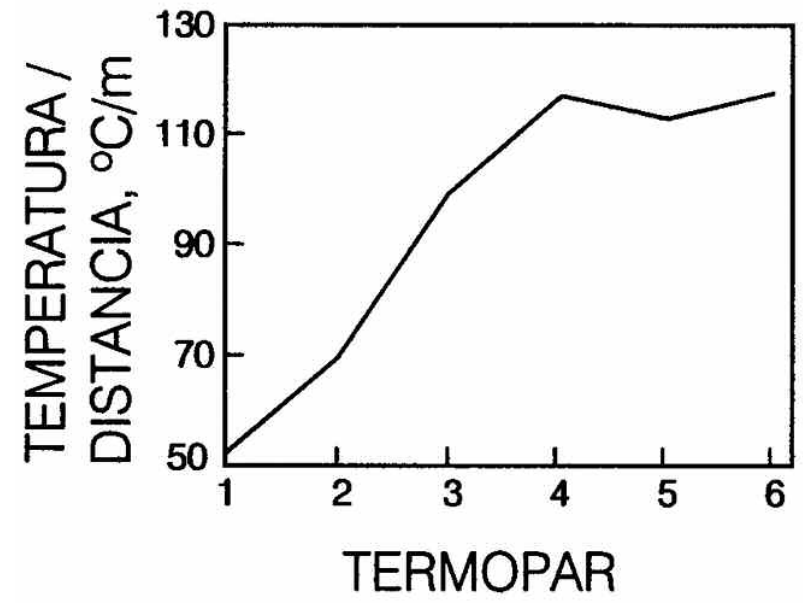

Figura 5. Variación de temperatura entre el termopar de las sondas sobre la carga y en la carga frente a la distancia entre el nivel de carga y el nivel de la sonda en la carga.

Figure 5. Temperature variation between thermocouple of above and in-burden probe versus the distance between burden level and in-burden probe level.

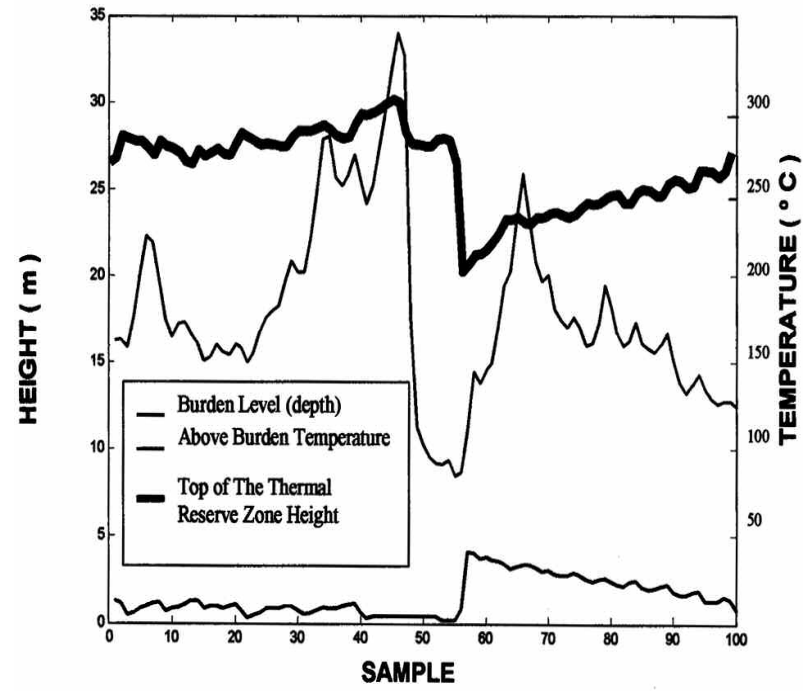

Figura 6. Estimación de la altura del límite superior de la zona de reserva térmica según el termopar 5 en la línea vertical.

Figure 6. Estimation of the top of the thermal reserve zone height at thermocouple 5 vertical line.

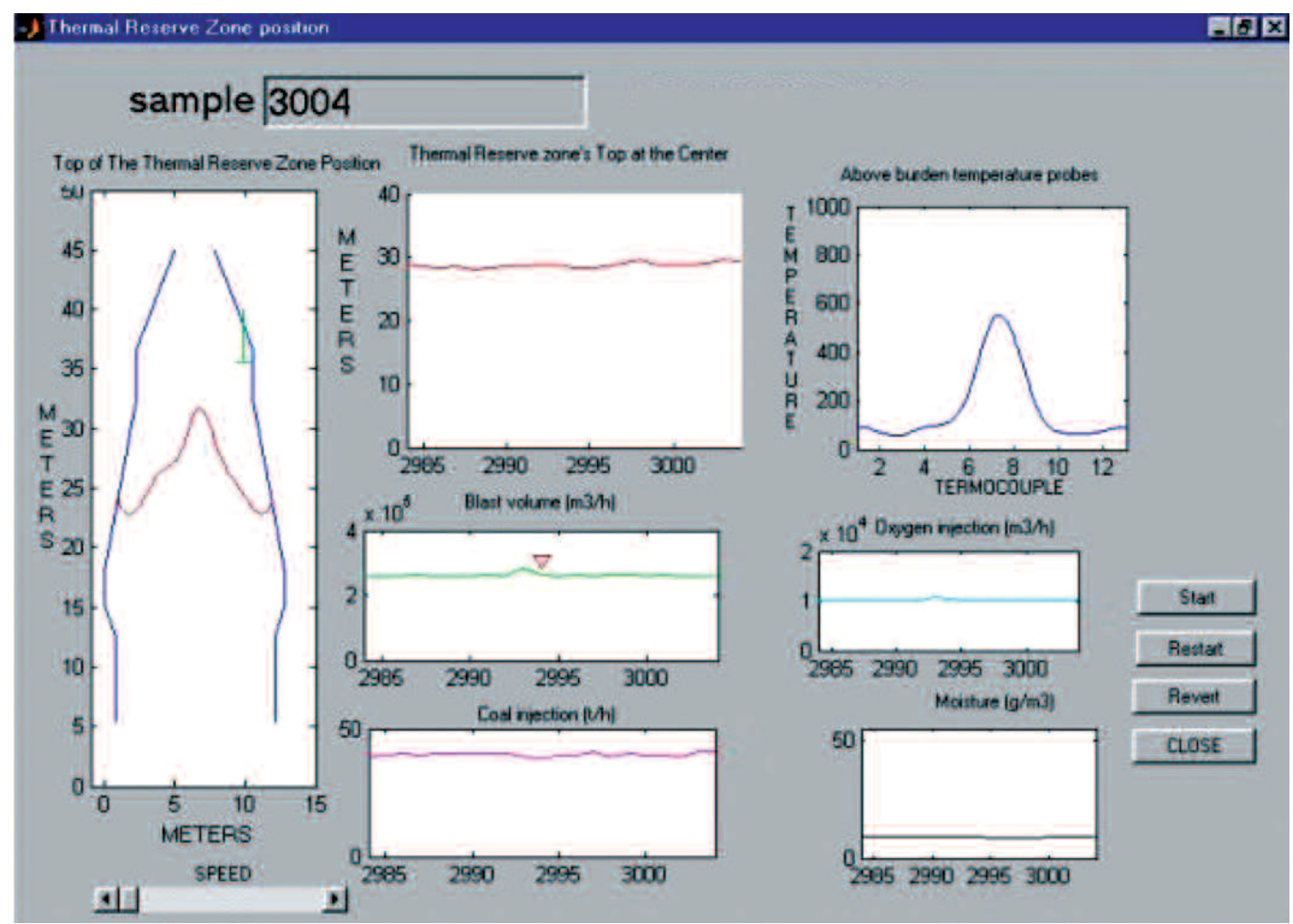

Figura 7. Pantalla de la interfase gráfica de usuario off-line de la posición de la zona de reserva térmica. Modo interrumpido.

Figure 7. Screen of the off-line graphic user interface of the thermal reserve zone position. Paused mode. 


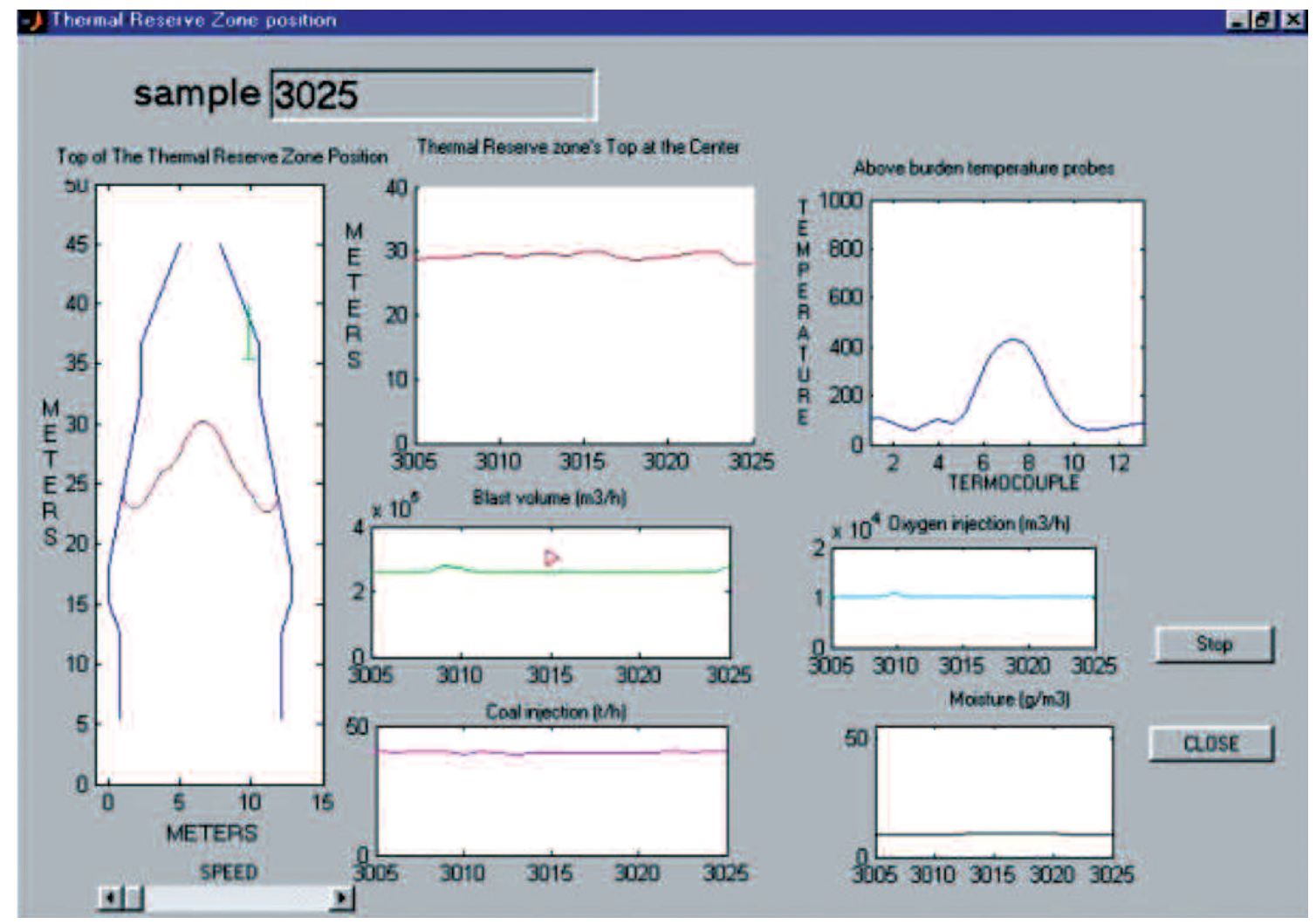

Figura 8. Pantalla de la interfase gráfica de usuario off-line de la posición de la zona de reserva térmica. Modo continuo.

Figure 8. Screen of the off-line graphic user interface of the thermal reserve zone position. Running mode.

sondas, sobre la carga y en la carga, para el cálculo del gradiente de temperaturas en la parte superior de la cuba, que permite deducir el límite superior de la zona de reserva térmica. La estimación indirecta de la zona cohesiva se puede justificar, por los resultados de las observaciones realizadas en hornos altos enfriados rápidamente, que muestran que la forma general de la zona cohesiva es similar a la forma de las isotermas que corresponden a la zona de reserva térmica (Fig. 1) [3 y 4].

Se ha desarrollado una interfase gráfica de usuario que estima la posición de la zona de reserva térmica y de otras variables destacadas. Los resultados obtenidos confirman que este sistema de operación ofrece al operador del horno alto una descripción valiosa del perfil del límite superior de la zona de reserva térmica y de su evolución con el tiempo ${ }^{[18]}$.

En la figura 10 se ofrece la forma y situación de las isotermas que limitan la zona cohesiva, tomando como referencia la zona de reserva térmica determinada experimentalmente, como se indica en la figura 2. La zona cohesiva presenta una altura de $(23,5-$ 14) $=9,5 \mathrm{~m}$.
En la parte más alta de la isoterma de $950^{\circ} \mathrm{C}$, que corresponde a la zona de reserva térmica, el gradiente de temperatura es del orden de $80^{\circ} \mathrm{C} / \mathrm{m}$, por lo cual la parte superior de la isoterma de $1.300^{\circ} \mathrm{C}$, se localiza a unos $4 \mathrm{~m}$ por debajo.

Teniendo en cuenta que en la parte más alta de la zona cohesiva el gradiente de temperatura es del orden de $120^{\circ} \mathrm{C} / \mathrm{m}$, la isoterma de fusión $\left(1.400^{\circ} \mathrm{C}\right)$ está, aproximadamente, $1 \mathrm{~m}$ por debajo de la isoterma de reblandecimiento $\left(1.300^{\circ} \mathrm{C}\right)$.

\section{CONCLUSIONES}

El trabajo realizado en planta piloto de sinterización y en horno alto sobre una estimación de la situación de la zona cohesiva y la zona de reserva térmica en el horno alto, permite establecer las conclusiones siguientes:

— Las medidas de temperaturas realizadas con sondas en el horno alto han permitido situar la posición de la isoterma de mayor temperatura de $950^{\circ} \mathrm{C}$, 


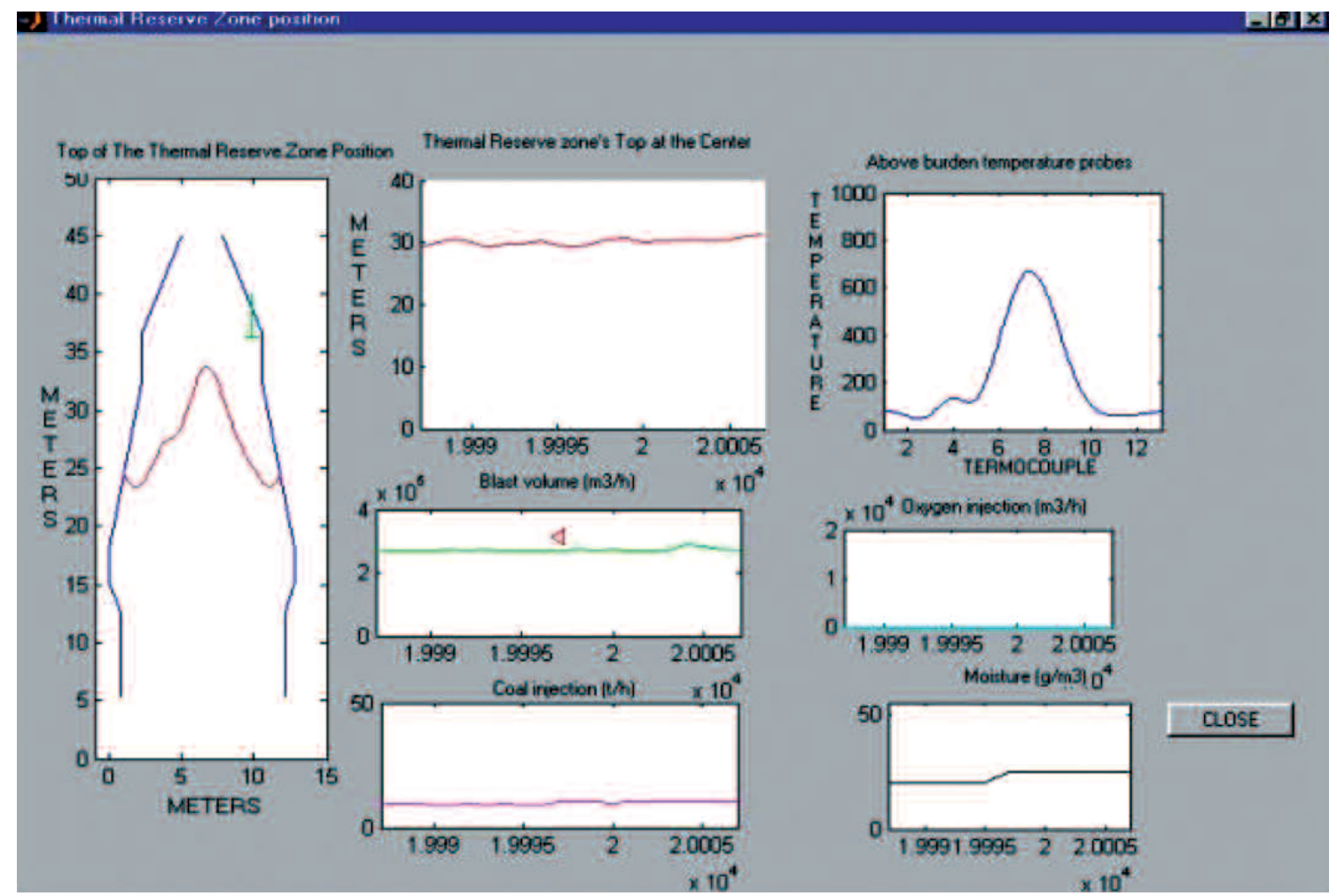

Figura 9. Pantalla de la interfase gráfica de usuario en línea de la posición de la zona de reserva térmica.

Figure 9. Screen of the on-line graphic user interface of the thermal reserve zone position.

Tabla VII. Parámetros de los hornos altos

Table VII. Blast furnace parameters

\begin{tabular}{lcccc}
\hline Horno alto & $\begin{array}{c}\text { Diámetro de } \\
\text { crisol, } \mathbf{m}\end{array}$ & $\begin{array}{c}\text { Volumen } \\
\text { interno, } \mathbf{m}^{\mathbf{3}}\end{array}$ & Altura, $\mathbf{m}$ & $\begin{array}{c}\text { Altura de la zona } \\
\text { cohesiva, } \mathbf{~ m}\end{array}$ \\
\hline Kukioka BF-4 & 7,9 & 1.407 & 31 & 9 \\
Hirohata BF-1 & 8,5 & 1.279 & 28 & 12 \\
Kokura BF-2 & 8,4 & 1.350 & 30 & 7 \\
Gijón HA-B & 11,3 & 2.349 & 36,7 & 9,5 \\
\hline
\end{tabular}

que corresponde a la zona de reserva térmica, localizada en la zona inferior de la cuba del horno. Estas medidas han permitido establecer una correlación entre las medidas realizadas con la sonda situada sobre la carga y las medidas realizadas con la sonda introducida en la carga.

- El tratamiento y evaluación de los datos de las variables principales medidas en el horno alto, ha permitido el desarrollo de un sistema de interfase gráfica de usuario, que ofrece al operador del horno una descripción valiosa del perfil del límite superior de la zona de reserva térmica y de su evolución con el tiempo.

- Se han fabricado, en planta piloto, una serie de sinterizados a partir de distintas mezclas minerales utilizadas en los hornos altos. Se ha determinado las temperaturas de reblandecimiento y fusión de estos sinterizados, lo que permite estimar los valores de las isotermas que limitan la zona cohesiva del horno, en el intervalo de $1.300-1.400{ }^{\circ} \mathrm{C}$.

- La zona cohesiva se extiende desde los etalajes del horno hasta la zona inferior de la cuba y tiene una altura de 9,5 m. 
SITUACIÓN ESTIMADA DE LA ZONA COHESIVA EN EL HORNO ALTO FORECAST SITUATION OF THE BLAST FURNACE COHESIVE ZONE

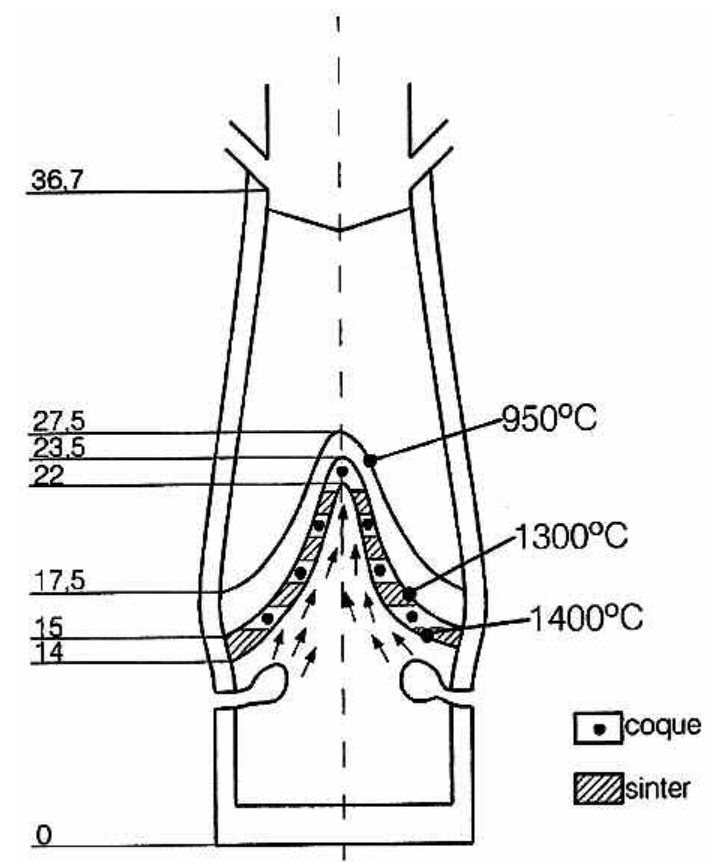

Figura 10. Isoterma del límite superior de la zona de reserva térmica e isotermas que delimitan la zona cohesiva. Cotas en metros.

Figure 10. Isotherm of the top limit of the thermal reserve zone and isotherms delimiting the cohesive zone. Height in meters.

\section{REFERENCIAS}

[1] A. Babich, D. Senk, H.W. Gudenau y K.Th. Mavrommatis, Ironmaking, Ed. RWTH Aachen University, Aachen, Alemania, 2008, pp. 402.

[2] N.J. Busby, T.A.T. Fray y D.C. Goldring, Ironmaking Steelmaking 21 (1994) 229-236.

[3] K. Kanbara, T. Hagiwara, A. Shigemi, S. Kondo, Y. Kanayama, K. Wakabayashi y N. Hiramoto, Trans. Iron Steel Inst. Jpn. 17 (1977) 371-380.
[4] M. Sasaki, K. Ono, A. Suzuki, Y. Okuno y K. Yoshizawa, Trans. Iron Steel Inst. Jpn. 17 (1977) 391-400.

[5] G. Clixby, Ironmaking Steelmaking 13 (1986) 169-175.

[6] D.C. Goldring, J.A. Jones y K. Grebe, Ironmaking Steelmaking 24 (1997) 373-385.

[7] M.A. Propster y J. Szekely, Ironmaking Steelmaking 6 (1979) 209-220.

[8] S. Wakamaya, Y. Kanamaya y Y. Okuno, Ironmaking Steelmaking 6 (1979) 262-267.

[9] A. Cores, A. Babich, M. Muñiz, A. Isidro, S. Ferreira y R. Martín, Ironmaking Steelmaking 34 (2007) 231-240.

[10] A. Formoso, A. Moro, G. Fernández-Pello, M. Muñiz, A. Moro y A. Cores, Rev. Metal. Madrid 36 (2000) 254-265.

[11] A. Formoso, A. Moro, G. Fernández-Pello, J.L. Menéndez, M. Muñiz y A. Cores, Ironmaking Steelmaking 30 (2003) 447-460.

[12] Standard test method for fusibility of coal and coal ash. Designation: D1857-68 (Reapproved 1980), ASTM, Pittsburg, PA, California, EE. UU., 1980.

[13] European Blast Furnace Commiittee, VDEh, Düsseldorf, Alemania, 2004.

[14] C.E. Loo, ICSTI Ironmaking Conf. Proc., 1998, pp. 1.299-1.316.

[15] K. Sasaki, F. Nakatani, M. Hatano, M. Watanabe, T. Shimoda, K. Yokotani, T. Ito y T. Yokoi, Trans. Iron Steel Inst. Jpn. 17 (1977) 252-261.

[16] L. Lu, R.J. Holmes y J.R. Manuel, ISIJ Int. 47 (2007) 349-358.

[17] M. Matsumura, M. Hoshi y T. Kawaguchi, ISIJ Int. 45 (2005) 594-602.

[18] D. Sert, G. Danloy, O. Havelange y J. Saiz de Ayala. ECSC contrato no $7210-\mathrm{PR} / 122$, Informe final, 2002. 